International Mathematical Forum, 2, 2007, no. 61, 3027 - 3040

\title{
Equivalence among Various Generalized Derivatives and Generalized Subdifferentials of Functions Defined on Binormed Spaces
}

\author{
S. Lahrech and A. Bouaicha \\ Dept of Mathematics, Faculty of science, Mohamed first University \\ Oujda, Morocco, (GAFO Laboratory)
}

\begin{abstract}
We take any binormed space $\left(E,\|\cdot\|_{1},\|\cdot\|_{2}\right)$ such that $\left(E,\|\cdot\|_{2}\right)$ is a Banach space and the norm $\|\cdot\|_{2}$ is finer than the norm $\|\cdot\|_{1}$.

We prove that if a real function $f$ is such that $-f$ is $\left(\|\cdot\|_{1},\|\cdot\|_{2}\right)$-locally Lipschitzian around $x \in E$ and regular at this point with respect to the norm $\|\cdot\|_{1}$, then $f$ is $\left(\|\cdot\|_{1},\|\cdot\|_{2}\right)$-strictly differentiable at $x$ iff it is $\|\cdot\|_{1}$-regular at $x$ iff it is Gâteaux differentiable at $x$ iff the generalized Clarke subdifferential $\partial_{0}^{1,2} f(x) \equiv \partial_{0}^{\|\cdot\|_{1},\|\cdot\|_{2}} f(x)$, The Michel-Penot subdifferential $\partial_{\diamond}^{2} f(x) \equiv \partial_{\diamond}^{\|\cdot\|_{2}} f(x)$, the modified upper Dini subdifferential $\partial^{D, 2} f(x)$ or the modified lower Dini subdifferential $\partial_{D}^{2} f(x)$ is a singleton iff the upper Dini subdifferential $\partial^{+, 2} f(x)$ or the lower Dini subdifferential $\partial^{-, 2} f(x)$ is non empty iff the upper Dini derivative $f^{+}(x,$.$) or the$ lower Dini derivative $f^{-}(x,$.$) is subadditive.$

Finally, we give an example which shows that our characterization of strict differentiability given in Theorem 12 is more general and more adapted for application than the one given by Zili Wu and J.J.Ye in [2].
\end{abstract}

Mathematics Subject Classification: 49J52, 49J50

Keywords: Strictly differentiable mappings, Generalized Clarke subdifferential, Dini and modified Dini subdifferentials, Regularity

\section{An Introduction to Generalized Clarke Sub- differentiability}

In this paragraph, we briefly review some basic notions and results established by Samir Lahrech and related to the generalized Clarke subdifferential in binormed spaces. For the detail, see [1]. 
We shall be working in binormed space $\left(E,\|\cdot\|_{1},\|\cdot\|_{2}\right)$ such that $\left(E,\|\cdot\|_{2}\right)$ is a Banach space and for some $c>0\|\cdot\|_{1} \leq c\|\cdot\|_{2}$.

Let $U$ be an open set of $\left(E,\|\cdot\|_{1}\right)$, and let $h_{2}$ be a $\|\cdot\|_{2}$-locally Lipschitz real function around $\bar{x} \in U$.

Let $v$ any vector in $E$. The Clarke generalized directional derivative of $h_{2}$ at $\bar{x}$ in the direction $v$ with respect to the norm $\|\cdot\|_{2}$, denoted $h_{2}^{0,2}(\bar{x}, v)$, is defined as follows:

$$
h_{2}^{0,2}(\bar{x}, v)=\limsup _{x^{\prime} \rightarrow\|\cdot\|_{2} \bar{x}, t \downarrow 0} \frac{h_{2}\left(x^{\prime}+t v\right)-h_{2}\left(x^{\prime}\right)}{t} .
$$

The Clarke subdifferential of $h_{2}$ at $\bar{x}$ with respect to the norm $\|\cdot\|_{2}$, denoted $\partial_{0}^{2} h_{2}(\bar{x})$, is the subset of $\left(E,\|\cdot\|_{2}\right)^{\prime}$ given by

$$
\partial_{0}^{2} h_{2}(\bar{x})=\left\{\xi \in\left(E,\|\cdot\|_{2}\right)^{\prime}: h_{2}^{0,2}(\bar{x}, v) \geq<\xi, v>\forall v \in E\right\} .
$$

According to [3], the generalized Clarke subdifferential of $h_{2}$ at $\bar{x}$ with respect to the pair of norms $\left(\|\cdot\|_{1},\|\cdot\|_{2}\right)$, denoted $\partial_{0}^{1,2} h_{2}(\bar{x})$, is the subset of $\left(E,\|\cdot\|_{2}\right)^{\prime}$ given by

$$
\partial_{0}^{1,2} h_{2}(\bar{x})=\left\{\xi \in\left(E,\|\cdot\|_{2}\right)^{\prime}: h_{2}^{0,1}(\bar{x}, v) \geq<\xi, v>\forall v \in E\right\} .
$$

Notice that the Clarke subdifferential $\partial_{0}^{2} h_{2}(\bar{x})$ is smaller than the generalized Clarke subdifferential $\partial_{0}^{1,2} h_{2}(\bar{x})$. More precisely, we have $\partial_{0}^{2} h_{2}(\bar{x}) \subset \partial_{0}^{1,2} h_{2}(\bar{x})$. If Moreover, $h_{2}$ is locally Lipschitz around $\bar{x}$ with respect to the norm $\|\cdot\|_{1}$, then $\partial_{0}^{2} h_{2}(\bar{x})=\partial_{0}^{1} h_{2}(\bar{x})=\partial_{0}^{1,2} h_{2}(\bar{x})$.

In particular, if we take $\|\cdot\|_{1}=\|\cdot\|_{2}$, then $\partial_{0}^{2} h_{2}(\bar{x})=\partial_{0}^{1} h_{2}(\bar{x})=\partial_{0}^{1,2} h_{2}(\bar{x})$.

So, the generalized Clarke subdifferential is a generalized version of the Clarke subdifferential.

Let us remark that $h_{2}^{0,1}(\bar{x},$.$) is the support function of the generalized Clarke$ subdifferential $\partial_{0}^{1,2} h_{2}(\bar{x})$.

\subsection{Topological Properties of Generalized Clarke Sub- differential}

Our goals in this paragraph is to give some topological properties of generalized Clarke subdifferential. For this, we introduce the notion of locally Lipschitzian mappings with respect to the pair of norms.

We say that $h_{2}$ is $\left(\|\cdot\|_{1},\|.\|_{2}\right)$ locally Lipschitzian around $\bar{x}$ if for some nonnegative scalar $K$, one has

$$
\left|h_{2}(y)-h_{2}\left(y^{\prime}\right)\right| \leq K\left\|y-y^{\prime}\right\|_{2}
$$

for all $y, y^{\prime}$ close to $\bar{x}$ with respect to the norm $\|.\|_{1}$.

The following summarizes some basic properties of the generalized Clarke subdifferential. 
proposition $1 \partial_{0}^{1,2} h_{2}(\bar{x})$ is a nonempty, convex, closed subset of $\left(\left(E,\|\cdot\|_{2}\right)^{\prime}, * \sigma\left(\left(E,\|\cdot\|_{2}\right)^{\prime}, E\right)\right)$. Moreover, if $h_{2}$ is $\left(\|\cdot\|_{1},\|\cdot\|_{2}\right)$ locally Lipschitzian around $\bar{x}$, then $\partial_{0}^{1,2} h_{2}(\bar{x})$ is weakly*-compact in $\left(\left(E,\|\cdot\|_{2}\right)^{\prime}, * \sigma\left(\left(E,\|\cdot\|_{2}\right)^{\prime}, E\right)\right)$.

Proof.

The set $\partial_{0}^{1,2} h_{2}(\bar{x})$ is nonempty, since $\partial_{0}^{2} h_{2}(\bar{x}) \subset \partial_{0}^{1,2} h_{2}(\bar{x})$. The convexity and the closedness of $\partial_{0}^{1,2} h_{2}(\bar{x})$ are trivially fulfilled.

Assume now that $h_{2}$ is $\left(\|.\|_{1},\|.\|_{2}\right)$ locally Lipschitzian around $\bar{x}$. Then, $\partial_{0}^{1,2} h_{2}(\bar{x})$ is bounded in $\left(\left(E,\|\cdot\|_{2}\right)^{\prime},\|\cdot\|_{\left(E,\|\cdot\|_{2}\right)^{\prime}}\right)$. Therefore, using the Alaoglu's Theorem, we deduce the weak*-compactness of $\partial_{0}^{1,2} h_{2}(\bar{x})$.

The assertion below reiterates that the pair of multifunctions $\left(\partial_{0}^{2} h_{2}, \partial_{0}^{1,2} h_{2}\right)$ is closed from $\left(U,\|\cdot\|_{1}\right)$ to $\left(\left(E,\|\cdot\|_{2}\right)^{\prime}, * \sigma\left(\left(E,\|\cdot\|_{2}\right)^{\prime}, E\right)\right)$ in the following sense: if $x_{i}$ and $\xi_{i}$ are sequences in $U$ and $\left(E,\|\cdot\|_{2}\right)^{\prime}$ such that $\xi_{i} \in \partial_{0}^{2} h_{2}\left(x_{i}\right), x_{i}$ converges to $x$ with respect to the norm $\left\|_{1}\right\|_{1}$, and $\xi_{i}$ converges to $\xi$ for the weak topology * $\sigma\left(\left(E,\|\cdot\|_{2}\right)^{\prime}, E\right)$, then $\xi \in \partial_{0}^{1,2} h_{2}(x)$.

proposition 2 The pair of multifunctions $\left(\partial_{0}^{2} h_{2}, \partial_{0}^{1,2} h_{2}\right)$ is closed from $\left(U,\|\cdot\|_{1}\right)$ to $\left(\left(E,\|\cdot\|_{2}\right)^{\prime}, * \sigma\left(\left(E,\|\cdot\|_{2}\right)^{\prime}, E\right)\right)$.

Proof. Let $x_{i}$ and $\xi_{i}$ be sequences in $U$ and $\left(E,\|.\|_{2}\right)^{\prime}$ such that $\xi_{i} \in \partial_{0}^{2} h_{2}\left(x_{i}\right)$, $x_{i}$ converges to $x$ with respect to the norm $\|.\|_{1}$, and $\xi_{i}$ converges to $\xi$ for the weak topology $* \sigma\left(\left(E,\|\cdot\|_{2}\right)^{\prime}, E\right)$.

Let any $v \in E$ be given. Then $\left\langle\xi_{i}, v>\right.$ converges to $<\xi, v>$. One has $h_{2}^{0,2}\left(x_{i}, v\right) \geq<\xi_{i}, v>$, which implies that $h_{2}^{0,1}\left(x_{i}, v\right) \geq<\xi_{i}, v>$.

By the upper semicontinuity of $h_{2}^{0,1}$ with respect to the norm $\|\cdot\|_{1}$, we deduce that $h_{2}^{0,1}(x, v) \geq<\xi, v>$. Since $v$ is arbitrary, $\xi$ belongs to $\partial_{0}^{1,2} h_{2}(x)$.

Remark 3 If $h_{2}$ is $\left(\|\cdot\|_{1},\|\cdot\|_{2}\right)$ locally Lipschitzian around $\bar{x}$. Then, $\zeta \in$ $\partial_{0}^{1,2} h_{2}(\bar{x})$ iff $h_{2}^{0,1}(\bar{x}, v) \geq<\zeta, v>\forall v \in E$.

\subsection{Relation to Taylor Derivatives And Generalized Sub- derivatives}

The main result of this section will be that if $h_{2}$ is $\left(\|\cdot\|_{1},\|.\|_{2}\right)$ locally Lipschitzian around $\bar{x}$, then $\partial_{0}^{1,2} h_{2}(\bar{x})$ is reduced to a singleton iff $h_{2}$ is $\left(\|\cdot\|_{1},\|\cdot\|_{2}\right)$ strictly Taylor differentiable at $\bar{x}$.

By $B_{1}$, respectively, $B_{2}$, we denote the unit ball in $\left(E,\|\cdot\|_{1}\right)$, respectively, the unit ball in $\left(E,\|\cdot\|_{2}\right)$.

Let $h$ a map acting from $U$ into a Banach space $(Y,\|\|$.$) , and let x \in U$.

We shall say that $h$ is $\left(\|\cdot\|_{1},\|\cdot\|_{2}\right)$-strictly Taylor differentiable at a point $x$ if there exists a continuous linear operator from $\left(E,\|.\|_{2}\right)$ to $(Y,\|\|$.$) denoted$ $\nabla h(x)$ such that for each $v$, the following holds:

$$
\lim _{x^{\prime} \rightarrow\|\cdot\|_{1} x, t \downarrow 0} \frac{h\left(x^{\prime}+t v\right)-h\left(x^{\prime}\right)}{t}=\nabla h(x) v,
$$


where the convergence is uniform for $v$ in compact sets in $\left(E,\|\cdot\|_{2}\right)$. Note that ours is a Hadamard-type strict derivative.

Let us remark that if $h$ is a $\left(\|\cdot\|_{1},\|\cdot\|_{2}\right)$-strictly Taylor differentiable at $x, h$ is not necessarily $\|\cdot\|_{1}$-strictly differentiable at $x$. But if $\|\cdot\|_{1}$ is equivalent to the norm $\|\cdot\|_{2}$, then $h$ is necessarily $\|\cdot\|_{1}$-strictly differentiable at $x$.

So the notion of strict differentiability in terms of Taylor strengthens and generalizes the elegant notion of Hadamard-strict differentiability.

proposition 4 Let $L$ be a continuous linear operator from $\left(E,\|.\|_{2}\right)$ to $(Y,\|\|$.$) ,$ and let $x \in U$. The following are equivalent:

(i) $h$ is $\left(\|\cdot\|_{1},\|.\|_{2}\right)$-strictly Taylor differentiable at $x$ and $\nabla h(x)=L$.

(ii) $h$ is $\left(\|\cdot\|_{1},\|\cdot\|_{2}\right)$ - locally Lipschitzian around $x$, and for each $v$ in $E$ one has

$$
\lim _{x^{\prime} \rightarrow\|\cdot\|_{1} x, t \downarrow 0} \frac{h\left(x^{\prime}+t v\right)-h\left(x^{\prime}\right)}{t}=L v .
$$

\section{Proof.}

Assume (i). The equality in (ii) holds by assumption, so to prove (ii) it suffices to show that $h$ is $\left(\|\cdot\|_{1},\|\cdot\|_{2}\right)$ - locally Lipschitzian around $x$. If this is not the case, there exist sequences $\left\{x_{n}\right\}$ and $\left\{x_{n}^{\prime}\right\}$ converging to $x$ with respect to the norm $\|\cdot\|_{1}$ such that $x_{n}, x_{n}^{\prime}$ lie in $x+\frac{1}{n} B_{1}$ and

$$
\left\|h\left(x_{n}\right)-h\left(x_{n}^{\prime}\right)\right\|>n\left\|x_{n}-x_{n}^{\prime}\right\|_{2} .
$$

Let us define $t_{n}>0$ and $v_{n}$ via $x_{n}^{\prime}=x_{n}+t_{n} v_{n}$ and $\left\|v_{n}\right\|_{2}=n^{-\frac{1}{2}}$. It follows that $t_{n} \rightarrow 0$.

Let $V$ consists of the points in the sequence $\left\{v_{n}\right\}$ together with 0 . Note that $V$ is compact in $\left(E,\|\cdot\|_{2}\right)$, so that by definition of $\nabla h(x)$ for any $\varepsilon$ there exists $n_{0}$ such that, for all $n \geq n_{0}$, for all $v \in V$, one has

$$
\left\|\frac{h\left(x_{n}+t_{n} v\right)-h\left(x_{n}\right)}{t_{n}}-\nabla h(x) v\right\|<\varepsilon .
$$

But this is impossible since when $v=v_{n}$, the term $\frac{h\left(x_{n}+t_{n} v\right)-h\left(x_{n}\right)}{t_{n}}$ has norm exceeding $n^{\frac{1}{2}}$ by construction. Thus, (ii) holds.

We now posit (ii). Let $V$ any compact subset of $\left(E,\|\cdot\|_{2}\right)$ and $\varepsilon$ any positive number. In view of (ii), there exists for each $v$ in $V$ a number $\delta(v)$ such that

$$
\left\|\frac{h\left(x^{\prime}+t v\right)-h\left(x^{\prime}\right)}{t}-\nabla h(x) v\right\|<\varepsilon
$$

for all $x^{\prime} \in x+\delta(v) B_{1}$ and $\left.t \in\right] 0, \delta[$. Since the norm of

$$
\frac{h\left(x^{\prime}+t v^{\prime}\right)-h\left(x^{\prime}\right)}{t}-\frac{h\left(x^{\prime}+t v\right)-h\left(x^{\prime}\right)}{t}
$$


is bounded above by $k\left\|v-v^{\prime}\right\|_{2}$ (where $K$ is a $\left(\|\cdot\|_{1},\|\cdot\|_{2}\right.$ )-Lipschitz constant for $h$ and where $x^{\prime}$ is sufficiently $\|.\|_{1}$ near $x$ and $t$ is sufficiently near 0 ), we deduce from the last inequality that for a suitable redefinition of $\delta(v)$, one has

$$
\left\|\frac{h\left(x^{\prime}+t v^{\prime}\right)-h\left(x^{\prime}\right)}{t}-L v^{\prime}\right\|<2 \varepsilon
$$

for all $x^{\prime}$ in $x+\delta(v) B_{1}, v^{\prime}$ in $v+\delta(v) B_{2}$, and $t$ in $] 0, \delta$. A finite number of the $\|\cdot\|_{2}$-open sets $\left\{v+\delta(v) B_{2}: v \in V\right\}$ will cover $V$, say, those that correspond to $v_{1}, \ldots, v_{n}$. If we set $\delta^{\prime}=\min _{1 \leq i \leq n} \delta\left(v_{i}\right)$, it follows then that

$$
\left\|\frac{h\left(x^{\prime}+t v^{\prime}\right)-h\left(x^{\prime}\right)}{t}-L v^{\prime}\right\|<2 \varepsilon
$$

for any $v \in V$, for all $x^{\prime} \in x+\delta^{\prime} B_{1}$ and $\left.t \in\right] 0, \delta^{\prime}\left[\right.$. Thus, $h$ is $\left(\|.\|_{1},\|\cdot\|_{2}\right)$ - strictly Taylor differentiable at $x$ and $\nabla h(x)=L$, and the proof is complete.

Now we can prove the main result of this section.

Theorem 5 Assume that $h_{2}$ is $\left(\|\cdot\|_{1},\|\cdot\|_{2}\right)$ locally Lipschitzian around $\bar{x}$. Then $\partial_{0}^{1,2} h_{2}(\bar{x})$ is reduced to a singleton $\{\zeta\}$ iff $h_{2}$ is $\left(\|\cdot\|_{1},\|.\|_{2}\right)$ strictly Taylor differentiable at $\bar{x}$. In this case, $\zeta=\nabla h_{2}(\bar{x})$.

In particular, if $\|\cdot\|_{1}=\|\cdot\|_{2}$ then $\partial_{0}^{1} h_{2}(\bar{x})$ is reduced to a singleton $\{\zeta\}$ iff $h_{2}$ is $\|\cdot\|_{1}$-strictly differentiable at $\bar{x}$.

\section{Proof.}

Suppose first that $h_{2}$ is $\left(\|\cdot\|_{1},\|.\|_{2}\right)$ strictly Taylor differentiable at $\bar{x}$, then $h_{2}^{0,1}(\bar{x}, v)=\nabla h_{2}(\bar{x}) v$. Therefore, $\partial_{0}^{1,2} h_{2}(\bar{x})$ is reduced to a singleton $\left\{\nabla h_{2}(\bar{x})\right\}$. To prove the converse, it suffices to show that the condition of Proposition 4 holds if $\partial_{0}^{1,2} h_{2}(\bar{x})$ is reduced to a singleton $\{\zeta\}$. We begin by showing that $h_{2}^{0,1}(\bar{x}, v)=<\zeta, v>$ for each $v$.

Let $v \in E$. (Note that $h_{2}^{0,1}(\bar{x}, v) \geq<\zeta, v>$ ). By the Hahn-Banach Theorem there exists $\zeta^{\prime} \in\left(E,\|.\|_{1}\right)^{\prime}$ majorized by $h_{2}^{0,1}(\bar{x},$.$) and agreeing with$ $h_{2}^{0,1}(\bar{x},$.$) at v$. Since $h_{2}$ is $\left(\|.\|_{1},\|\cdot\|_{2}\right)$ locally Lipschitzian around $\bar{x}$, then $\zeta^{\prime} \in\left(E,\|\cdot\|_{2}\right)^{\prime}$. It follows then that $\zeta^{\prime} \in \partial_{0}^{1,2} h_{2}(\bar{x})$. Consequently, $\zeta=\zeta^{\prime}$. Therefore, $h_{2}^{0,1}(\bar{x}, v)=\langle\zeta, v>$.

We now calculate:

$$
\begin{aligned}
& \liminf _{x^{\prime} \rightarrow\|\cdot\|_{1} \bar{x}, t \downarrow 0} \frac{h_{2}\left(x^{\prime}+t v\right)-h_{2}\left(x^{\prime}\right)}{t}=-\limsup _{x^{\prime} \rightarrow\|\cdot\|_{1} \bar{x}, t \downarrow 0} \frac{h_{2}\left(x^{\prime}\right)-h_{2}\left(x^{\prime}+t v\right)}{t} \\
& =-\lim _{x^{\prime} \rightarrow\|\cdot\|_{1} \bar{x}, t \downarrow 0} \frac{h_{2}\left(x^{\prime}+t v-t v\right)-h_{2}\left(x^{\prime}+t v\right)}{t} \\
& =-h_{2}^{0,1}(\bar{x},-v) \\
& =-\langle\zeta,-v\rangle
\end{aligned}
$$




$$
\begin{aligned}
& =\langle\zeta, v> \\
& =h_{2}^{0,1}(\bar{x}, v) \\
& =\limsup _{x^{\prime} \rightarrow\|\cdot\|_{1}} \frac{h_{2}\left(x^{\prime}+t v\right)-h_{2}\left(x^{\prime}\right)}{t} .
\end{aligned}
$$

This establishes the limit condition of Proposition 4 and completes the proof.

\section{Characterization of $\left(\|\cdot\|_{1},\|\cdot\|_{2}\right)$-strictly differ- entiable mappings via various subdifferen- tials}

Assume that all the hypothesis of paragraph 1 are satisfied. Let $f$ be a real function defined on $E$. Assume that $f$ is $\left(\|.\|_{1},\|\cdot\|_{2}\right)$-locally Lipschitzian around $x \in E$.

We briefly review some basic notions we will use.

- The Clarke derivative of $f$ at $x$ in the direction $v$ with respect to the norm $\|.\|_{1}$ is

$$
f^{0,1}(x, v):=\limsup _{x^{\prime} \rightarrow\|\cdot\|_{1}} \overline{x, t \downarrow 0} \frac{f\left(x^{\prime}+t v\right)-f\left(x^{\prime}\right)}{t} .
$$

- The Michel-Penot derivative of $f$ at $x$ in the direction $v$ is

$$
f^{\diamond}(x, v):=\sup _{y} \limsup _{t \rightarrow 0^{+}} \frac{f(x+t y+t v)-f(x+t y)}{t} .
$$

- The upper Dini derivative of $f$ at $x$ in the direction $v$ is

$$
f^{+}(x, v):=\limsup _{t \rightarrow 0^{+}} \frac{f(x+t v)-f(x)}{t}
$$

while the lower Dini derivative of $f$ at $x$ in the direction $v$ is

$$
f^{-}(x, v):=\liminf _{t \rightarrow 0^{+}} \frac{f(x+t v)-f(x)}{t} .
$$

- The modified upper Dini derivative of $f$ at $x$ in the direction $v$ is

$$
f^{D}(x, v):=\sup _{u \in E}\left\{f^{+}(x, v+u)-f^{+}(x, u)\right\}
$$

while the modified lower Dini derivative of $f$ at $x$ in the direction $v$ is

$$
f_{D}(x, v):=\sup _{u \in E}\left\{f^{-}(x, v+u)-f^{-}(x, u)\right\} .
$$


Denote

$$
\partial^{\wedge, 2} f(x):=\left\{\xi \in\left(E,\|\cdot\|_{2}\right)^{\prime}: f^{\wedge}(x, v) \geq<\xi, v>, \forall v \in E\right\} .
$$

By replacing $f^{\wedge}$ with $f^{\diamond}, f^{+}, f^{-}, f^{D}$, and $f_{D}$ in this pattern we can define the Michel-Penot subdifferential $\partial^{\diamond, 2} f(x)$, the upper Dini subdifferential $\partial^{+, 2} f(x)$, the lower Dini subdifferential $\partial^{-, 2} f(x)$, the modified upper Dini subdifferential $\partial^{D, 2} f(x)$, and the modified lower Dini subdifferential $\partial_{D}^{2} f(x)$ of $f$ at $x$.

It is easy to see that for each $v \in E$ we have

$$
f^{-}(x, v) \leq f^{+}(x, v) \leq f^{D}(x, v) \leq f^{\diamond}(x, v) \leq f^{0,1}(x, v)
$$

and

$$
f^{-}(x, v) \leq f_{D}(x, v) \leq f^{\diamond}(x, v) \leq f^{0,1}(x, v) .
$$

Therefore the above subdifferentials have the following relationships:

$$
\partial^{-, 2} f(x) \subset \partial^{+, 2} f(x) \subset \partial^{D, 2} f(x) \subset \partial^{\diamond, 2} f(x) \subset \partial_{0}{ }^{1,2} f(x)
$$

and

$$
\partial^{-, 2} f(x) \subset \partial_{D}^{2} f(x) \subset \partial^{\diamond, 2} f(x) \subset \partial_{0}{ }^{1,2} f(x) .
$$

Note that $f^{+}(x,$.$) and f^{-}(x,$.$) are positive homogeneous. If they are subaddi-$ tive then $\partial^{+, 2} f(x)$ and $\partial^{-, 2} f(x)$ must be nonempty. In this case $\partial^{+, 2} f(x)$ and $\partial^{-, 2} f(x)$ coincide with $\partial^{D, 2} f(x)$ and $\partial_{D}^{2} f(x)$, respectively.

proposition 6 Let $f: E \longrightarrow]-\infty,+\infty\left[\right.$ be a $\left(\|\cdot\|_{1},\|\cdot\|_{2}\right)$-locally Lipschitzian mapping around $x$. Then

(a) $f^{+}(x,$.$) is subadditive iff \partial^{+, 2} f(x)=\partial^{D, 2} f(x)$;

(b) $f^{-}(x,$.$) is subadditive iff \partial^{-, 2} f(x)=\partial_{D}^{2} f(x)$.

\section{Proof.}

Since $f$ is $\left(\|\cdot\|_{1},\|\cdot\|_{2}\right)$-locally Lipschitzian around $x$, then it is $\|\cdot\|_{2}$-locally Lipschitzian near $x$. Therefore, by (Proposition 2.1, [2]), we deduce the result.

proposition 7 Let $f$ and $g$ be two $\left(\|\cdot\|_{1},\|\cdot\|_{2}\right)$-locally Lipschitz functions around $x \in E$. Then

$$
\partial_{0}^{1,2}(f+g)(x) \subset \partial_{0}^{1,2} f(x)+\partial_{0}^{1,2} g(x) .
$$

Proof.

To derive the result, it suffices to prove that

$$
(f+g)^{0,1}(x, .) \leq f^{0,1}(x, .)+g^{0,1}(x, .)
$$


since $f^{0,1}(x,$.$) and g^{0,1}(x,$.$) are respectively the support functions of the gen-$ eralized Clarke subdifferentials $\partial_{0}^{1,2} f(x)$ and $\partial_{0}^{1,2} g(x)$.

Let $v \in E$. Then

$$
\begin{gathered}
(f+g)^{0,1}(x, v)=\limsup _{\substack{x^{\prime} \rightarrow \|_{\|} x \\
t \rightarrow 0^{+}}} \frac{f\left(x^{\prime}+t v\right)+g\left(x^{\prime}+t v\right)-f\left(x^{\prime}\right)-g\left(x^{\prime}\right)}{t} \\
\leq \limsup _{\substack{x^{\prime} \rightarrow\|\cdot\|_{1} \\
t \rightarrow 0^{+}}} \frac{f\left(x^{\prime}+t v\right)-f\left(x^{\prime}\right)}{t}+\limsup _{\substack{x^{\prime} \rightarrow\|\cdot\|_{1} x \\
t \rightarrow 0^{+}}} \frac{g\left(x^{\prime}+t v\right)-g\left(x^{\prime}\right)}{t} \\
\leq f^{0,1}(x, v)+g^{0,1}(x, v) .
\end{gathered}
$$

Hence,

$$
\partial_{0}^{1,2}(f+g)(x) \subset \partial_{0}{ }^{1,2} f(x)+\partial_{0}{ }^{1,2} g(x) .
$$

Thus, we achieve the proof.

Recall that a $\left(\|\cdot\|_{1},\|\cdot\|_{2}\right)$-locally Lipschitz function $f$ defined on $E$ is said to be regular at $x \in E$ with respect to the norm \|\|$_{1}$ if for all $v \in E$, the usual one-sided directional derivative

$$
f^{\prime}(x, v)=\lim _{t \rightarrow 0^{+}} \frac{f(x+t v)-f(x)}{t} \text { exists and equals } f^{0,1}(x, v) .
$$

This means that if $f$ is a $\left(\|\cdot\|_{1},\|\cdot\|_{2}\right)$-locally Lipschitz function around $x$, and if moreover, $f$ is regular at $x$ with respect to the norm $\|\cdot\|_{1}$, then the generalized Clarke subdifferential $\partial_{0}{ }^{1,2} f(x)$ is reduced to the subdifferential $\partial^{2} f(x)$ in the sense of convex analysis given by

$$
\partial^{2} f(x)=\left\{\xi \in\left(E,\|.\|_{2}\right)^{\prime}: f^{\prime}(x, v) \geq<\xi, v>\forall v \in E\right\} .
$$

proposition 8 Let $f$ and $g$ be two $\left(\|\cdot\|_{1},\|\cdot\|_{2}\right)$-locally Lipschitz functions around $x \in E$. Assume that $f$ and $g$ are regular at $x$ with respect to the norm $\|.\|_{1}$. Then

$$
\partial_{0}^{1,2}(f+g)(x)=\partial_{0}^{1,2} f(x)+\partial_{0}^{1,2} g(x) .
$$

Proof.

By Proposition 7, it suffices to show that

$$
(f+g)^{0,1}(x, .) \geq f^{0,1}(x, .)+g^{0,1}(x, .) .
$$

Let $v \in E$. We have

$$
(f+g)^{0,1}(x, v)=\limsup _{\substack{x^{\prime} \rightarrow\|\cdot\|_{1} x \\ t \rightarrow 0^{+}}} \frac{f\left(x^{\prime}+t v\right)+g\left(x^{\prime}+t v\right)-f\left(x^{\prime}\right)-g\left(x^{\prime}\right)}{t}
$$




$$
\begin{gathered}
\geq \limsup _{t \rightarrow 0^{+}} \frac{f(x+t v)+g(x+t v)-f(x)-g(x)}{t} \\
\geq \liminf _{t \rightarrow 0^{+}} \frac{f(x+t v)-f(x)}{t}+\limsup _{t \rightarrow 0^{+}} \frac{g(x+t v)-g(x)}{t}=f^{\prime}(x, v)+g^{\prime}(x, v) .
\end{gathered}
$$

Therefore, since $f^{\prime}(x, v)=f^{0,1}(x, v)$ and $g^{\prime}(x, v)=g^{0,1}(x, v)$, we deduce that

$$
(f+g)^{0,1}(x, v) \geq f^{0,1}(x, v)+g^{0,1}(x, v),
$$

from which

$$
(f+g)^{0,1}(x, v)=f^{0,1}(x, v)+g^{0,1}(x, v) .
$$

Hence,

$$
\partial_{0}^{1,2}(f+g)(x)=\partial_{0}^{1,2} f(x)+\partial_{0}^{1,2} g(x) .
$$

It is known by Proposition 4 and Theorem 5 that a real function $f$ defined on $E$ is $\left(\|\cdot\|_{1},\|\cdot\|_{2}\right)$-strictly differentiable at $x$ iff $f$ is $\left(\|.\|_{1},\|.\|_{2}\right)$-locally Lipschitzian at $x$ and the generalized Clarke subdifferential $\partial_{0}{ }^{1,2} f(x)$ is a singleton. Applying this fact to a $\left(\|\cdot\|_{1},\|\cdot\|_{2}\right)$-locally Lipschitz function, we obtain the following characterization of the $\left(\|\cdot\|_{1},\|\cdot\|_{2}\right)$-strict differentiability.

proposition 9 Let $f$ be a $\left(\|\cdot\|_{1},\|\cdot\|_{2}\right)$-locally Lipschitz function around $x \in E$. Then $f$ is $\left(\|\cdot\|_{1},\|\cdot\|_{2}\right)$-strictly differentiable at $x$ iff both $f$ and $-f$ are $\|.\|_{1}$ regular at $x$.

Proof.

Assume that $f$ is $\left(\|\cdot\|_{1},\|\cdot\|_{2}\right)$-strictly differentiable at $x$, then

$$
\lim _{x^{\prime} \rightarrow\|\cdot\|_{1} x, t \downarrow 0^{+}} \frac{f\left(x^{\prime}+t v\right)-f\left(x^{\prime}\right)}{t}=\nabla f(x) \cdot v=\lim _{t \downarrow 0^{+}} \frac{f(x+t v)-f(x)}{t}=f^{\prime}(x, v),
$$

which means that

$$
f^{0,1}(x, v)=f^{\prime}(x, v) .
$$

Thus, $f$ is $\|\cdot\|_{1}$-regular at $x$. Using the same argument, we deduce that $-f$ is also $\|.\|_{1}$-regular at $x$.

Conversely, if $f$ and $-f$ are both $\|\cdot\|_{1}$-regular at $x$, then by Proposition 8 we have

$$
\{0\}=\partial_{0}{ }^{1,2}(f-f)(x)=\partial_{0}{ }^{1,2} f(x)+\partial_{0}{ }^{1,2}(-f)(x) .
$$

Consequently, $\partial_{0}{ }^{1,2} f(x)$ is a singleton. Hence, by Theorem 5 , we conclude that $f$ is $\left(\|\cdot\|_{1},\|\cdot\|_{2}\right)$-strictly differentiable at $x$.

To derive more characteristics for the $\left(\|\cdot\|_{1},\|\cdot\|_{2}\right)$-strict differentiability of a $\left(\|\cdot\|_{1},\|\cdot\|_{2}\right)$-locally Lipschitz function $f$, we need the next two propositions.

proposition 10 Let $f: E \longrightarrow]-\infty,+\infty\left[\right.$ be a $\left(\|\cdot\|_{1},\|\cdot\|_{2}\right)$-locally Lipschitz function around $x$. Then $f$ is $\|\cdot\|_{1}$-regular at $x$ iff $f^{-}(x,$.$) is subadditive and$ $\partial_{D}{ }^{2} f(x)=\partial_{0}{ }^{1,2} f(x)$. 


\section{Proof.}

By definition, $f$ is $\|\cdot\|_{1}$-regular at $x$ iff $f^{-}(x, v)=f^{0,1}(x, v)$ for each $v \in E$, which is equivalent to $\partial^{-, 2} f(x)=\partial_{D}^{2} f(x)=\partial_{0}{ }^{1,2} f(x)$.

But, according to Proposition 6 , this holds if and only if $f^{-}(x,$.$) is subadditive$ and $\partial_{D}^{2} f(x)=\partial_{0}{ }^{1,2} f(x)$.

proposition 11 Let $f: E \longrightarrow]-\infty,+\infty\left[\right.$ be a $\left(\|\cdot\|_{1},\|\cdot\|_{2}\right)$-locally Lipschitz function around $x$ such that $-f$ is $\|.\|_{1}$-regular at $x$. Then $\partial^{-, 2} f(x)=\partial^{+, 2} f(x)$ and $\partial_{D}{ }^{2} f(x)=\partial^{D, 2} f(x)=\partial_{\diamond}{ }^{2} f(x)=\partial_{0}{ }^{1,2} f(x)$.

\section{Proof.}

Let $v \in E$. Since $f$ is $\left(\|\cdot\|_{1},\|\cdot\|_{2}\right)$-Locally Lipschitzian around $x$ and $-f$ is $\|\cdot\|_{1^{-}}$ regular at $x$, then $(-f)^{\prime}(x, v)$ exists and equals $(-f)^{0,1}(x, v)$ for each $v \in E$. Thus, for each $v \in E, f^{\prime}(x, v)$ exists, and hence $f^{-}(x, v)=f^{+}(x, v)$ from which it follows that $\partial^{-, 2} f(x)=\partial^{+, 2} f(x)$.

Since for all $v \in E$ there hold the following inequalities:

$$
(-f)^{\prime}(x, v) \leq(-f)^{D}(x, v) \leq(-f)^{\diamond}(x, v) \leq(-f)^{0,1}(x, v)
$$

and

$$
(-f)^{\prime}(x, v) \leq(-f)_{D}(x, v) \leq(-f)^{\diamond}(x, v) \leq(-f)^{0,1}(x, v),
$$

then we obtain that

$$
(-f)^{\prime}(x, v)=(-f)^{D}(x, v)=(-f)_{D}(x, v)=(-f)^{\diamond}(x, v)=(-f)^{0,1}(x, v) .
$$

Moreover, we have

$$
f^{D}(x,-v)=(-f)_{D}(x, v) \text { and } f^{0,1}(x,-v)=(-f)^{0,1}(x, v) .
$$

Hence,

$$
f^{D}(x, v)=(-f)_{D}(x,-v)=(-f)^{0,1}(x,-v)=f^{0,1}(x, v)
$$

and

$$
f_{D}(x, v)=(-f)^{D}(x,-v)=(-f)^{0,1}(x,-v)=f^{0,1}(x, v) .
$$

Therefore,

$$
\partial^{D, 2} f(x)=\partial_{0}{ }^{1,2} f(x) \text { and } \partial_{D}{ }^{2} f(x)=\partial_{0}{ }^{1,2} f(x) .
$$

These two equalities imply that

$$
\partial^{D, 2} f(x)=\partial_{D}^{2} f(x)=\partial_{\diamond}^{2} f(x)=\partial_{0}{ }^{1,2} f(x)
$$

holds since

$$
\partial^{D, 2} f(x) \cup \partial_{D}{ }^{2} f(x) \subseteq \partial_{\diamond}{ }^{2} f(x) \subseteq \partial_{0}{ }^{1,2} f(x) .
$$

Thus, we achieve the proof.

With the above propositions it easy to prove that if $-f$ is $\left(\|\cdot\|_{1},\|\cdot\|_{2}\right)$-locally Lipschitzian around $x$ and $\|\cdot\|_{1}$-regular at $x$, then the $\left(\|\cdot\|_{1},\|\cdot\|_{2}\right)$-strict differentiability, the $\|\cdot\|_{1}$-regularity and the Gâteaux differentiability of $f$ at $x$ are all equivalent. 
Theorem 12 Let $f$ be a $\left(\|\cdot\|_{1},\|.\|_{2}\right)$-locally Lipschitz function around $x$ such that $-f$ is $\|\cdot\|_{1}$-regular at $x$. Then the following conditions are equivalent: (a) $f$ is $\left(\|\cdot\|_{1},\|\cdot\|_{2}\right)$-strictly differentiable at $x$;

(b) $f$ is $\|.\|_{1}$-regular at $x$;

(c) $f$ is Gâteaux differentiable at $x$;

(d) $\partial_{0}{ }^{1,2} f(x), \partial_{\diamond}{ }^{2} f(x), \partial^{D, 2} f(x)$, or $\partial_{D}{ }^{2} f(x)$ is a singleton ;

(e) $\partial^{+, 2} f(x)$ or $\partial^{-, 2} f(x)$ is nonempty;

(f) $f^{+}(x,$.$) or f^{-}(x,$.$) is subadditive.$

In particular, if the norm $\|\cdot\|_{1}$ coincides with the norm $\|\cdot\|_{2}$, then our result is equivalent to the result established by Zili Wu and J.J.Ye (see [2]).

\section{Proof.}

We prove this theorem in the following route:

$$
(a) \Rightarrow(b) \Rightarrow(f) \Rightarrow(e) \Rightarrow(c) \Rightarrow(d) \Rightarrow(a) \text {. }
$$

$(a) \Rightarrow(b)$ is direct from Proposition 9 .

Since $-f$ is $\|.\|_{1}$-regular at $x$, then for each $v \in E(-f)^{\prime}(x, v)$ exists and hence $f^{\prime}(x, v)$ exists, i.e., $f^{-}(x, v)$ coincides with $f^{+}(x, v)$. Hence, if $f$ is $\|$. $\|_{1}$-regular at $x$ then, by Proposition 10 , both $f^{-}(x,$.$) and f^{+}(x,$.$) are subadditive. There-$ fore, $(b) \Rightarrow(f)$.

$(f) \Rightarrow(e)$ follows directly from Proposition 6 and the fact that both $\partial^{D, 2} f(x)$ and $\partial_{D}{ }^{2} f(x)$ are nonempty.

To show $(e) \Rightarrow(c)$, since by Proposition 11, $\partial^{-, 2} f(x)=\partial^{+, 2} f(x)$, we only need to prove that the nonemptiness of $\partial^{-, 2} f(x)$ implies the Gâteaux differentiability of $f$ at $x$. But this is obvious as long as we note that $f$ is Gâteaux differentiable at $x$ iff both $\partial^{-, 2} f(x)$ and $\partial^{-, 2}(-f)(x)$ are nonempty and that $\partial^{-, 2}(-f)(x)=\partial_{0}{ }^{1,2}(-f)(x)$ from the $\|\cdot\|_{1}$-regularity of $-f$ at $x$.

$(c) \Rightarrow(d)$ follows from Proposition 11 and the fact that $\partial_{\diamond}{ }^{2} f(x)$ is a singleton when $f$ is Gâteaux differentiable at $x$.

$(d) \Rightarrow(a)$ is also immediate from Theorem 5 . Thus, the proof is complete.

Let us give now an example which shows that our characterization of strict differentiability given in theorem 12 is more general and more adapted for application than the one given by Zili $\mathrm{Wu}$ and J.J.Ye in [2].

Example 13 Let $\Omega$ a bounded domain in $R^{2}, E=W_{0}^{1,2}(\Omega)$ the Sobolev space with the usual norm $\|\cdot\|_{2}=\|\cdot\|_{W_{0}^{1,2}(\Omega)}$. Let also $p$ and $\varepsilon$ such that $0<\varepsilon<1$, $\varepsilon+2<p<\infty$. Set $\|\cdot\|_{1}=\|\cdot\|_{L^{p}(\Omega)}$. Remark that $\left(E,\left\|_{\cdot}\right\|_{2}\right)$ is a Banach separable space.

Since $W_{0}^{1,2}(\Omega) \hookrightarrow L^{p}(\Omega)$ then $\left(E,\|.\|_{1},\|.\|_{2}\right)$ is a binormed space such that for some $c>0\|\cdot\|_{1} \leq c\|\cdot\|_{2}$.

Set $g(u)=|u|^{\varepsilon+2}$ and consider the functional $G$ defined on $E$ by

$$
G(x)=\int_{\Omega} g(x(s)) d s .
$$


Then $G$ is $\|\cdot\|_{2}$-twice Fréchet differentiable at every $x \in E$ and

$$
\begin{aligned}
G^{(1)}(x) h & =\int_{\Omega} g^{\prime}(x(s)) h(s) d s \\
G^{(2)}(x)\left(h_{1}, h_{2}\right) & =\int_{\Omega} g^{\prime \prime}(x(s)) h_{1}(s) h_{2}(s) d s .
\end{aligned}
$$

We assert that $G$ is $\left(\|\cdot\|_{1},\|\cdot\|_{2}\right)$ - Fréchet strictly differentiable at every $x \in E$. Indeed, suppose by the contrary that there exists $x \in E, \varepsilon>0, x_{m}^{\prime} \in E, h_{m} \in E$ such that $x_{m}^{\prime} \rightarrow x$ in $\left(E,\|\cdot\|_{1}\right), h_{m} \rightarrow 0$ in $\left(E,\|\cdot\|_{1}\right)$ and $\left|r\left(x_{m}^{\prime}, h_{m}\right)\right|>\varepsilon\left\|h_{m}\right\|_{2}$, where

$$
r\left(x^{\prime}, h\right)=G\left(x^{\prime}+h\right)-G\left(x^{\prime}\right)-G^{(1)}(x) h .
$$

Then,

$$
\left|r\left(x_{m}^{\prime}, h_{m}\right)\right|=\left|\int_{\Omega} \int_{0}^{1}\left[g^{\prime}\left(x_{m}^{\prime}(s)+t h_{m}(s)\right)-g^{\prime}(x(s))\right] h_{m}(s) d t d s\right| .
$$

Let $p^{\prime}>1$ such that $\frac{1}{p}+\frac{1}{p^{\prime}}=1$. Since $p>\varepsilon+2$ then $p>p^{\prime}(\varepsilon+1)$. Thus, $x_{m}^{\prime} \rightarrow x$ in $\left(E,\|\cdot\|_{L^{p^{\prime}(\varepsilon+1)}(\Omega)}\right)$ and $h_{m} \rightarrow 0$ in $\left(E,\|\cdot\|_{L^{p^{\prime}(\varepsilon+1)}(\Omega)}\right)$. Without loss of generality we can suppose that there exists $Z_{1} \in L^{p^{\prime}(\varepsilon+1)}(\Omega)$ such that almost everywhere in $\Omega$

$$
\left|x_{m}^{\prime}(s)\right|+\left|h_{m}(s)\right| \leq Z_{1}(s), h_{m}(s) \rightarrow 0, x_{m}^{\prime}(s) \rightarrow x(s) .
$$

Using the Holder inequality, we deduce that

$$
\left|r\left(x_{m}^{\prime}, h_{m}\right)\right| \leq\left[\int_{\Omega} \int_{0}^{1}\left|g^{\prime}\left(x_{m}^{\prime}(s)+t h_{m}(s)\right)-g^{\prime}(x(s))\right|^{p^{\prime}} d t d s\right]^{\frac{1}{p^{\prime}}}\left\|h_{m}\right\|_{L^{p}(\Omega)} .
$$

Consequently,

$$
\left.\left|r\left(x_{m}^{\prime}, h_{m}\right)\right| \leq c\left[\int_{\Omega} \int_{0}^{1}\left|g^{\prime}\left(x_{m}^{\prime}(s)+t h_{m}(s)\right)-g^{\prime}(x(s))\right|^{p^{\prime}} d t d s\right]\right]^{\frac{1}{p^{\prime}}}\left\|h_{m}\right\|_{2} .
$$

But this contradicts the fact that $\left|r\left(x_{m}^{\prime}, h_{m}\right)\right|>\varepsilon\left\|h_{m}\right\|_{2}$ since

$$
\int_{\Omega} \int_{0}^{1}\left|g^{\prime}\left(x_{m}^{\prime}(s)+t h_{m}(s)\right)-g^{\prime}(x(s))\right|^{p^{\prime}} d t d s \rightarrow 0
$$

by the dominated convergence theorem.

Hence, $G$ is $\left(\|\cdot\|_{1},\|\cdot\|_{2}\right)$ - Fréchet strictly differentiable at every $x \in E$. This implies that $G$ is $\left(\|.\|_{1},\|.\|_{2}\right)$-Hadamard strictly differentiable at every $x \in E$, from which it follows that for each $v \in E, G$ and $-G$ are both $\|\cdot\|_{1}$-regular at $x$ and $\left(\|\cdot\|_{1},\|\cdot\|_{2}\right)$-Locally Lipschitzian around $x$. Therefore, by Theorem 12, we deduce that for every $x \in E$

$$
\partial_{0}{ }^{1,2} G(x)=\partial_{\diamond}{ }^{2} G(x)=\partial^{D, 2} G(x)=\partial_{D}{ }^{2} G(x)=\{\nabla G(x)\}
$$


Let us remark that in our case, the $\left(\|.\|_{1},\|.\|_{2}\right)$-Hadamard strictly differentiability is equivalent to the $\left(\|\cdot\|_{1},\|.\|_{2}\right)$-Fréchet strictly differentiability since $\left(W_{0}^{1,2}(\Omega),\|\cdot\|_{2}\right)$ is a reflexive Banach space.

Note that $G$ is not $\|.\|_{1}$-Fréchet differentiable at any point $x \in E$ and hence $G$ is not $\|\cdot\|_{1}$-Hadamard differentiable at any point $x \in E$. Indeed, let $\alpha_{m} \rightarrow \infty$ and $d_{m} \rightarrow \infty$ such that $\left|g\left(d_{m}\right)\right| \geq \alpha_{m}\left|d_{m}\right|^{p}$. By the countable additivity of the Lebesgue measure

$\exists C>0 \exists \Omega^{\prime} \subset \Omega$ such that $\mu\left(\Omega^{\prime}\right)>0$, $\operatorname{dist}\left(\Omega^{\prime}, \partial \Omega\right)>0$ and $\forall s \in \Omega^{\prime}|x(s)| \leq C$.

In this case, put $D=\max \{g(u):|u| \leq C\}<\infty$. Choose $\Omega_{m} \subset \Omega^{\prime}$ such that $\mu\left(\Omega_{m}\right)=\left|d_{m}\right|^{-p}\left|\alpha_{m}\right|^{-\frac{1}{2}}$ for large $m$.

Let $h_{m}$ defined by

$$
h_{m}(s)=\left\{\begin{array}{l}
d_{m}-x(s), s \in \Omega_{m}, \\
0, s \in \Omega \backslash \Omega_{m} .
\end{array}\right.
$$

It follows then that $\left\|h_{m}\right\|_{1} \rightarrow 0$ and

$\left|G\left(x+h_{m}\right)-G(x)\right| \geq \alpha_{m}\left|d_{m}\right|^{p} \mu\left(\Omega_{m}\right)-D \mu\left(\Omega_{m}\right)=\left|\alpha_{m}\right|^{\frac{1}{2}}-D \mu\left(\Omega_{m}\right) \rightarrow+\infty$.

Let $k_{m} \in C_{0}^{\infty}(\Omega)$ such that $\left\|k_{m}-h_{m}\right\|_{1} \rightarrow 0$. Then $\left\|k_{m}\right\|_{1} \rightarrow 0$, but $G\left(x+k_{m}\right)-$ $G(x) \rightarrow \infty$ since the Lebesgue integral is absolutely continuous. Therefore, $G$ is not $\|.\|_{1}$-Fréchet differentiable at $x$, from which it follows that $G$ is not $\|\cdot\|_{1}$-Fréchet strictly differentiable at $x$. Thus, $G$ is not $\|.\|_{1}$-Hadamard strictly differentiable at $x$. Consequently, since $G$ is $\left(\|\cdot\|_{1},\|\cdot\|_{2}\right)$-Hadamard strictly differentiable at $x$, we deduce that $G$ is not $\|.\|_{1}$-locally Lipschitzian around $x$. Therefore, the characterization of the $\|.\|_{1}$-Hadamard strict differentiability given by Zili Wu and J.J.Ye in [2] cannot be used in our example. So, our characterization of strict differentiability given in theorem 12 is more general and more adapted for application than the one given by Zili Wu and J.J.Ye in [2].

\section{References}

[1] S.Lahrech, On the Topological Properties of the Generalized Clarke Subdifferential. Submitted to Acta Mathematica universitatis Comenianae.

[2] Zili Wu, J.J.Ye, Equivalence among various derivatives and subdifferentials of the distance functions. Journal of Mathematical Analysis And Applications. 282(2003) 629-647.

[3] S.Lahrech, A.Benbrik, On the Mordukhovich Subdifferential In Binormed Spaces and Some Applications. International Journal of Pure and Applied Mathematics. Volume 20, No.1, 2005, 31-39. 
[4] Jonathan M. Borwein, Adrian S. Lewis, Convex Analysis and Nonlinear Optimization. Canadian Mathematical Society, 2000.

[5] F.H. Clarke, Generalized gradients and applications. Transactions of the American Mathematical Society, 205:247-262, 1975.

[6] F.H. Clarke, Optimization and Nonsmooth Analysis. Wiley, New York, 1983.

[7] F.H. Clarke, Y.S. Ledyaev, R.J. Stern, P.R. Wolenski. Nonsmooth Analysis and control theory. Springer-Verlag, New York, 1998.

[8] P. Michel and J.-P. Penot. Calcul sous-différentiel pour les fonctions Lipschitziennes et non Lipschitziennes. Comptes Rendus de l'Académie des Sciences de Paris, 298:269-272, 1984.

Received: May 25, 2007 